\title{
Enhanced expression of $\alpha 2,3$-linked sialic acids promotes gastric cancer cell metastasis and correlates with poor prognosis
}

\author{
LI SHEN ${ }^{1,2}$, ZHIGUO LUO $^{1,2}$, JUNBO WU $^{3}$, LI QIU $^{1}$, MING LUO $^{1}$, QING KE $^{1,2}$ and XIAOXIA DONG ${ }^{4}$ \\ ${ }^{1}$ Department of Clinical Oncology, ${ }^{2}$ Institute of Cancer Research, ${ }^{3}$ Center for Evidence-based Medicine and Clinical Research, \\ Taihe Hospital, ${ }^{4}$ Department of Pharmacology, School of Basic Medical Sciences, \\ Hubei University of Medicine, Shiyan, Hubei 442000, P.R. China
}

Received October 17, 2016; Accepted December 12, 2016

DOI: $10.3892 /$ ijo.2017.3882

\begin{abstract}
Gastric cancer (GC) is a highly metastatic disease and one of the leading causes of cancer death in the world. Aberrant glycosylation is one of many molecular changes that accompany malignant transformation. This study was aimed at identification of glycan profiling changes in GC progression and its potential mechanisms. We employed a microarray with 91 lectins to compare the differential glycans in the three human GC cell lines, SGC-7901, BGC-823 and MGC-803. According to glycan-binding specificities of lectins, all GC cell lines expressed common sugar structures, such as mannose, galactose and fucose. Importantly, we found that the binding of Maackia amurensis lectin-I (MAL-I) to GC cells was proportional to their metastatic capacity. Further analysis revealed that the level of $\alpha 2,3$-linked sialic acids ( $\alpha 2-3 \mathrm{Sia})$, which can be recognized by MAL-I, was significantly overexpressed in MGC-803 cells, while low expression was detected in SGC-7901 cells. In addition, the mRNA and protein expression levels of $\beta$-galactoside $\alpha 2,3$-sialyltransferase IV (ST3Gal-IV), which was related to the synthesis of $\alpha 2-3 \mathrm{Sia}$, were substantially increased in MGC-803 cells. Knockdown of ST3Gal-IV in MGC-803 cells led to a decreased level of $\alpha 2-3$ Sia and decreased ability of invasion and migration. Exogenous expression of ST3Gal-IV in SGC-7901 cells enhanced cell migration, invasion and the content of $\alpha 2-3$ Sia. Furthermore, the staining of MAL-I in GC tissues showed that high expression of $\alpha 2-3$ Sia was closely correlated with lymph node metastasis, TNM stage and poor overall survival. These findings lead to better understanding of the function of $\alpha 2-3 \mathrm{Sia}$ in the progression and metastasis of GC. This property may be important for developing new therapeutic approaches for GC.
\end{abstract}

Correspondence to: Dr Xiaoxia Dong, Department of Pharmacology, School of Basic Medical Sciences, Hubei University of Medicine, 30 South Renmin Road, Shiyan, Hubei 442000, P.R. China

E-mail: xiaoxia.28@163.com

Key words: $\alpha 2,3$-sialic acids, ST3Gal-IV, gastric cancer, metastasis, glycosylation

\section{Introduction}

As one of the most common cancers, gastric cancer (GC) has the third highest lethality and fourth highest morbidity of all cancers worldwide (1). According to the GloboCan statistics, GC was responsible for $\sim 951,600$ new cancer cases and more than 723,000 deaths in 2012 (2). However, over half of these cases and deaths were estimated to occur in China. It is predicted that there will be $\sim 498,000$ cancer mortalities in 2015, ranking as the second most frequent cause of cancer death in China (3). The high mortality is caused by tumor recurrence, metastasis and lack of effective therapeutic response. Therefore, increasing efforts have been devoted to investigating the mechanisms of GC progression, especially the mechanisms of GC metastasis (4).

Cell surface glycans play important roles in intercellular and intracellular processes, including cell adhesion and development, cell recognition, and cancer development and metastasis (5). Mounting evidence suggests that glycans can affect tumor cell invasiveness, including the ability to disseminate through the circulation and metastasise into distant organs (6). The roles of glycans in cancer have been highlighted by the fact that alterations in glycosylation could promote invasive behaviour of tumor cells that ultimately lead to the progression of cancer (7). For example, metastatic progression of prostate cancer was mediated by aberrant $\mathrm{O}$-glycosylation of cancer cell surface receptors (8). N-glycans with bisecting GlcNAc shed light on the detection of ovarian cancer in early peritoneal metastasis stage (9). We therefore wished to discover novel glycans associated with the metastasis of GC.

Researchers have attempted to identify abnormal glycans involved in the malignancy of GC. For example, a global decrease in core-fucosylation of N-linked glycans was found to be correlated with the malignancy of GC patients $(10,11)$. However, the fluctuation and variation of glycans in GC are still largely unknown. High level of $\alpha 2,3$-linked sialic acids ( $\alpha 2-3 \mathrm{Sia})$ was associated with metastatic potential of $\mathrm{GC}$ cells (12). Nevertheless, the role of $\alpha 2-3$ Sia and its potential mechanisms in GC has not been as clearly defined. Recently, we utilized a microarray with 91 lectins to compare the differential glycans between the two GC cell lines with different metastatic ability. Importantly, we discovered significant 
connections between altered $\alpha 2-3 \mathrm{Sia}$ and the tumorigenesis, progression and metastasis of GC. Our results provide evidence that $\alpha 2$-3Sia could be an important molecular marker to determine malignant properties and a target for molecular therapy in patients with GC.

\section{Materials and methods}

Cell culture. Human GC cell lines, SGC-7901, BGC-823 and MGC-803 were purchased from the cell library of the Chinese Academy of Sciences (Shanghai, China). Both were cultured in RPMI-1640 medium with 10\% FBS (Gibco-BRL, Grand Island, NY, USA) at $37^{\circ} \mathrm{C}$ and $5 \% \mathrm{CO}_{2}$.

All procedures performed in this study involving human participants were in accordance with the ethical standards of Hubei University of Medicine and with the 1964 Helsinki declaration and its later amendments or comparable ethical standards.

Lectin microarray screening for cell surface glycans. The lectin microarray with 91 lectins was obtained from BC Biotechnology (Guangdong, China). The cells were harvested by trypsin digestion and fluorescently labeled with CFDA-SE (Life Technologies, Carlsbad,CA, USA). Then cells were resuspended in incubation solution (PBS with $1 \% \mathrm{BSA}, 0.5 \mathrm{mM} \mathrm{CaCl}{ }_{2}$ and $0.1 \mathrm{mM} \mathrm{MnCl} \mathrm{M}_{2}$ ). After blocked with $1 \%$ BSA, the lectin microarray was incubated with cells for $1 \mathrm{~h}$ at dark. Unbound cells were removed by washing buffer (PBS with $0.5 \%$ Tween). The signals were detected by a GenePix 4200A scanner and the fluorescence intensity of each spot was measured with GenePix Pro 6.0 software (Molecular Devices, Sunnyvale, CA, USA). The signal intensities are the averages of duplicate samples from three separate experiments.

Plasmid construction and transfection. Human $\beta$-galactoside $\alpha 2,3$-sialyltransferase IV (ST3Gal-IV) genetic fragment was amplified and introduced into the pEGFP-C1 eukaryotic expression vector (Clontech, Heidelberg, Germany). Primers 5' and $3^{\prime}$ for PCR were 5'-CGGATCCatggtcagcaagtccegctgg-3' and 5'-TCGCTCGAGtgttgtaggcgtctgggt-3'. The plasmid was synthesized by GenePharma (Shanghai, China). After being confirmed by XhoI and BamHI digestion analyses and DNA sequencing, the recombinant pEGFP-C1-ST3Gal-IV plasmid was transfected into cells. Transfection was carried out using Lipofectamine 3000 (Invitrogen, Carlsbad, CA, USA), according to the manufacturer's instructions. As a control, cells were transduced with a construct lacking ST3Gal-IV. Transfection efficiency was detected by fluorescence microscopy (Zeiss, Gottingen, Germany).

siRNA transfection. Human ST3Gal-IV and negative siRNA (control) were synthesized by GenePharma. The siRNA sequences targeting ST3Gal-IV were as follows: sence, 5'-GAC CCAGAAAUAAUCCUCAdTdT-3' and antisence, 5'-UGA GGAUUAUUUCUGGGUCdTdT-3'. The negative siRNA sequences were as follows: sense, 5'-uucuccGaacGuGucac GudTdT-3' and antisense, 5'-acGuGacacGuucGGaGaadTdT-3' Cells were transfected with ST3Gal-IV siRNA or negative siRNA using Lipofectamine 3000 as suggested by the manufacturer.
Table I. Sequences of the primers used for quantitative RT-PCR.

\begin{tabular}{ll}
\hline Primer name & \multicolumn{1}{c}{ Sequences (5'-3') } \\
\hline ST3Gal-I & F: ACCACCGACCTGCAGTGGGT \\
& R: GGGGGCACGGGGACATAGGT \\
ST3Gal-II & F: TCCGATTCACCTATGCCCCAGT \\
& R: TGCTCGGTCCACCTGTCGTGA \\
ST3Gal-III & F: CCGCTGGACAAACCCTAGGCAC \\
& R: GGCTAGCTCGGCAGGCAGTTT \\
ST3Gal-IV & F: ACCTATGAGCTGCCCTTTGGGACT \\
& R: GACACTCGAGGCTCTTTATGCTCTC \\
ST3Gal-V & F: TCAGTCAAGGTTCTGGGGCCGA \\
& R: CCCGCCAAACTGACTTCATCGCA \\
ST3Gal-VI & F: GACCTTCGAGACATATTTCAGCTCG \\
& R: TCCATTACCAACCACCACACACCT \\
GADPH & F: CCAACCGCGAGAAGATGA \\
& R: CCAGAGGCGTACAGGGATAG
\end{tabular}

$\mathrm{F}$, forward; $\mathrm{R}$, reverse.

RT-PCR and western blot analysis. Methods of RNA extraction and real-time RT-PCR analysis were conducted as previously described (13). Primer pairs for PCR are listed in Table I. Each sample was detected three times and expression of target genes was analyzed using the comparative threshold cycle $(\mathrm{Ct})$ method using GADPH as the reference gene. The total protein was gathered and the ST3Gal-IV and GAPDH antibodies (Santa Cruz Biotechnology, Inc., Santa Cruz, CA, USA) were used in the western blotting. The band intensity was developed using the ECL detection systems (Thermo Fisher Scientific, Waltham, MA, USA).

Wound healing assay for cell migration. Cells were seeded in a 6-well plate at a density of $5 \times 10^{5}$ cells/well and grown to confluence overnight. After transfected with ST3Gal-IV siRNA or pEGFP-C1-ST3Gal-IV plasmid for $24 \mathrm{~h}$, cells were incubated with serum-free medium. A micro-pipette tip was used to scratch a line in the cell monolayer. Following incubation for $24 \mathrm{~h}$, cell migration was observed and photographs were taken under a light microscope (Olympus BX41; Olympus Corporation, Tokyo, Japan).

Transwell assay for cell invasion. Cell invasion assay was performed using Transwell chamber $(8.0 \mu \mathrm{m}$ pore size; Costar, Cambridge, MA, USA). Cells were transfected with ST3Gal-IV siRNA or pEGFP-C1-ST3Gal-IV plasmid for $24 \mathrm{~h}$. Then cells were resuspended and added to the upper chamber of Matrigelcoated inserts. The lower chamber was filled with medium containing 10\% FBS. After $24 \mathrm{~h}$ incubation, cells on the upper surface were removed by gently scrubbing with a cotton swab. Cells that had migrated below the membrane were stained with eosin staining solution (Beyotime, Jiangsu, China).

Lectin blot. SDS-PAGE and electrophoretic transferring were performed in the same manner as described for western 
A

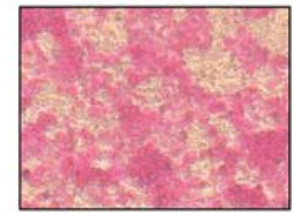

MGC-803

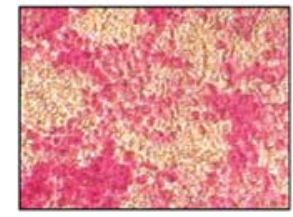

BGC-823

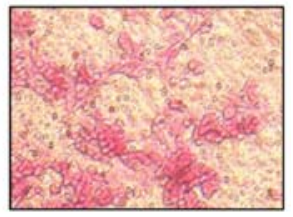

SGC-7901

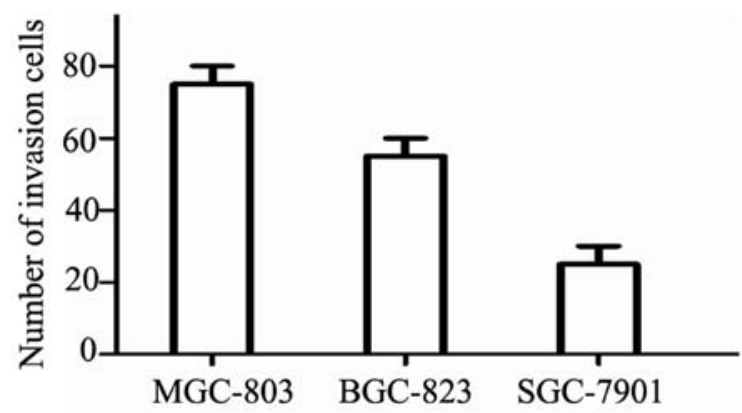

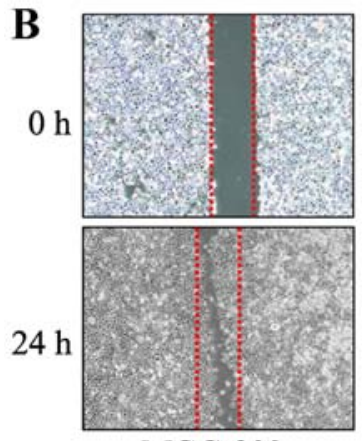

MGC-803
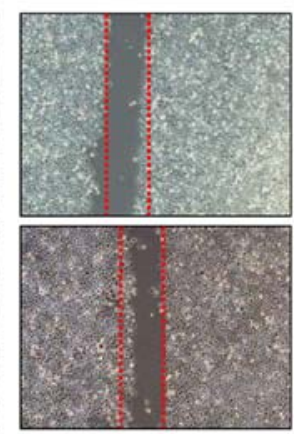

BGC-823

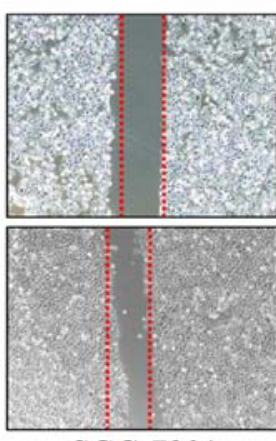

SGC-7901

Figure 1. Transwell and wound-healing assays were carried out to compare the invasive and migratory capabilities of GC cells. (A) The invasive ability was evaluated by counting the number of cells that had invaded the Matrigel and the 8 - $\mu$ m-pore Transwell membrane. (B) The migratory ability was evaluated with a wound-healing assay.

blot analysis. After blocking with carbo-free blocking solution the membrane was incubated with 1:1,000 dilution of biotin-labeled Maackia amurensis lectin I (MAL-I) (both from Vector Laboratories, Burlingame, CA, USA) for $2 \mathrm{~h}$ at room temperature. The blots were developed using ECL detection system.

Analysis of lectin labeling by flow cytometry. Cells were collected and stained with $10 \mu \mathrm{g} / \mathrm{ml}$ FITC-MAL-I (Vector Laboratories) at $25^{\circ} \mathrm{C}$ for $1 \mathrm{~h}$, then washed three times with PBS. Fluorescent analyses were performed using a FACScan flow cytometer (Becton-Dickinson, Mountain View, CA, USA).

Lectin histochemistry. From 2008 to 2014, 80 GC tissues were obtained from patients who underwent surgery at Taihe Hospital, Hubei University of Medicine. No patients received neoadjuvant chemotherapy or radiotherapy. The main clinical and pathologic variables are summarized in Table III. Paraffinembedded tissue sections were first deparaffinized, followed by blocking with $30 \%$ normal donkey serum. Biotinylated lectin was then added onto the slide $(50 \mu \mathrm{g} / \mathrm{ml})$ and incubated for $1 \mathrm{~h}$, followed by incubation with horseradish peroxidase (HRP)-conjugated streptavidin (Sigma, St. Louis, MO, USA). The following experimental procedure was according to the manufacturer's instructions of the DAB staining kit (Maixin Bio, Fuzhou, China). MAL-I staining intensities were analyzed by two observers independently using the semiquantitative immunoreactivity scoring (IRS) system as previously described (14). The IRS value $>4$ was defined as high expression and IRS value $\leq 4$ as low expression.

Statistical analysis. All data are expressed as mean \pm SD. Statistical analysis was carried out using SPSS 16.0 (SPSS, Inc., Chicago, IL, USA). Student's t-test was performed for comparisons between two groups. Correlations between MAL-I staining and clinicopathological parameters were evaluated by Chi-square test or Fisher's exact tests. Kaplan-Meier method was applied to compare overall survival (OS) for groups of low and high expression. A value of $\mathrm{P}<0.05$ was considered significant.

\section{Results}

Comparison of the metastatic ability of GC cells. Wound healing and Transwell assays were performed to compare the metastatic abilities of cultured GC cells. As shown in Fig. 1A, the number of MGC-803 cells that migrated into the lower chamber was much higher than the number of SGC-7901 cells. The distance between wound edges of MGC-803 cells 
A

\begin{tabular}{|c|c|c|c|c|c|}
\hline LTL & Jacalin & PSA & LCA & GSL I & UEA I \\
\hline AAL & CPA & LAL & TL & PHA-M & MAA \\
\hline SNA & SSA & ECA & MAL I & SNA-I & PHA-P \\
\hline NPL & HAA & ConA & LBA & GNL & PHA-E+L \\
\hline PTL II & HHL & CALSEPA & VRA & AMA & MNA-M \\
\hline Ricin B Chain & VFA & SJA & VGA & VVA mannose & ASA \\
\hline GSL II & UEA-II & BDA & LEL & STL & UDA \\
\hline PWM & TKA & WGA & DSL & HPA & PMA \\
\hline VVL & DBA & SBA & PTL I & SNA-II & WFA \\
\hline CSA & CAA & Black bean crude & LcH & GSL-IA4 & SHA \\
\hline IRA & IAA & HMA & MIA & GHA & MNA-G \\
\hline LPA & RPA & RCA I & LFA & PTA GalNAc & GSL I-B4 \\
\hline LcH B & PTA galactose & EEL & LAA & PNA & BPL \\
\hline PEA & ABA & AIA & ACL & MPL & PHA-L \\
\hline MOA & CA & PHA-E & RTA & ECL & MAL II \\
\hline PAA & - & - & $0.5 \%$ BSA & CY3 & CY5 \\
\hline
\end{tabular}

B

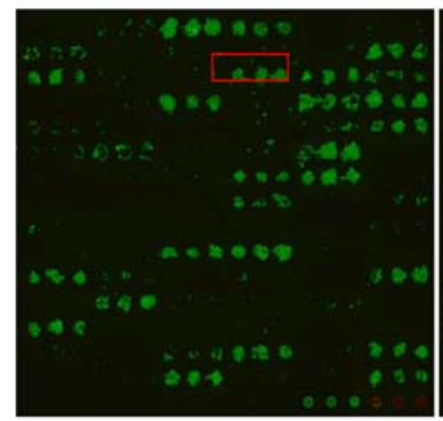

MGC-803

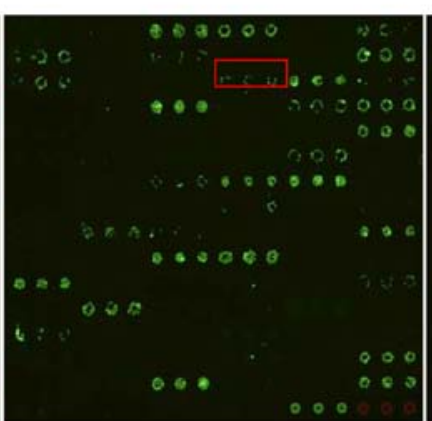

BGC-823

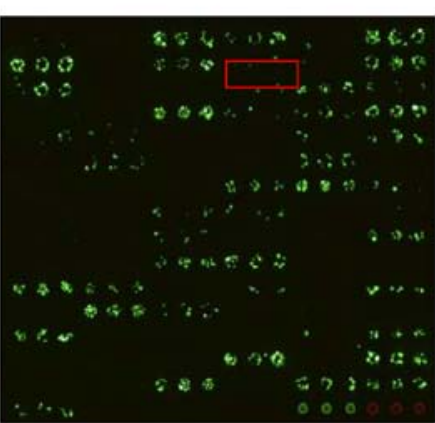

SGC-7901

C
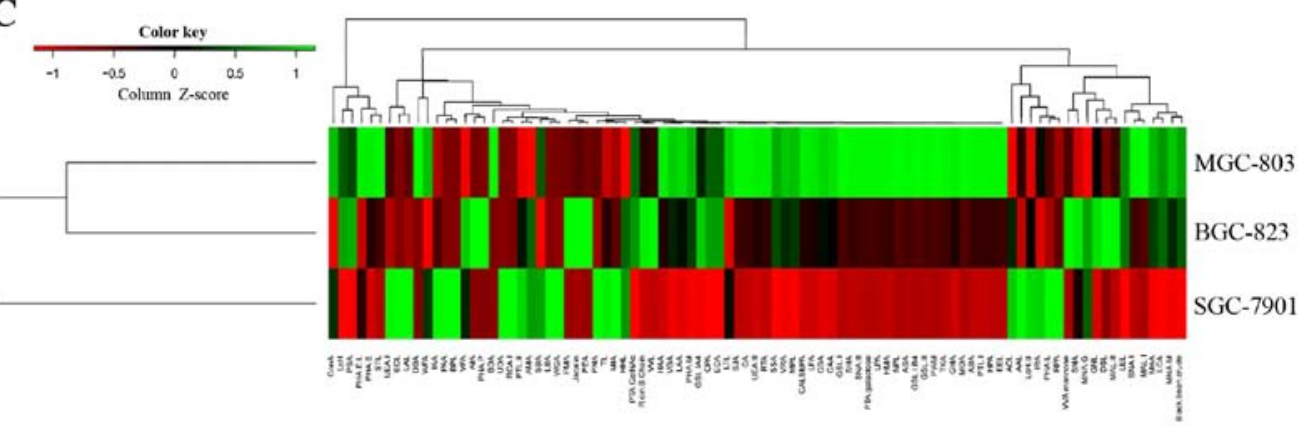

Figure 2. Identification of metastasis-specific lectin binding to GC cells. (A) Layout of the lectin microarray containing 91 lectins. (B) Representative lectin microarray binding patterns of the three GC cell lines. (C) Clustered heat map of the lectin cell-binding profiles.

was markedly shorter than that of SGC-7901 cells (Fig. 1B). MGC-803 cells had the highest invasive and migratory capabilities, SGC-7901 cells had the lowest, while BGC-823 cells had moderate capability.

Metastasis-specific lectin binding identified on GC cells. To identify the cell surface glycan profiles, freshly harvested cells were incubated on a lectin microarray containing 91 lectins, in which each lectin was present in triplicate. The lectin microarray format used in this study is shown in Fig. 2A. For live cell labeling, CFDA-SE reagents were used, which were converted to fluorescent derivatives once inside the cells. Using this analysis, a cell binding map was generated (Fig. 2B). For those lectins that showed no binding activity to the tested cells, it is possible that they either have relatively low affinity or are of low quality. We found that all cell lines expressed common sugar structures, such as mannose, galactose, and fucose, which are generally found on all mammalian cell surfaces. For example, the binding of all cell lines to the lectins HHL, specific for mannose, and
PNA, specific for terminal galactose was observed. To better compare the overall glycan profiles, a heat map was generated and clustered according to the lectin-binding pattern and intensity (Fig. 2C). Despite the similarities, there were still significant differences in lectin binding patterns among cultured GC cells. Overall, 8 lectins showed a strong binding capacity in MGC-803 cells, while 2 lectins strongly bound to SGC-7901 cells (Table II). It suggests that the cell lines with different metastatic potential still possess variations in their cell surface glycan signatures and can be captured by the lectin microarray. In addition, three metastasis-specific lectins LEL (Lycopersicon esculentum), Jacalin (Artocarpus integrifolia), and MAL-I showed the largest differences in fluorescent intensities. Each of these lectins effectively captured MGC-803 cells but weakly captured SGC-7901 cells. The most significant difference was seen for MAL-I. MAL-I is highly specific for $\alpha 2-3 \mathrm{Sia}$ (15). It means that MGC-803 cells predominantly express $\alpha 2-3 \mathrm{Sia}$. Collectively, the above findings indicate that the overexpression of $\alpha 2-3$ Sia may play an important role in GC metastasis. 
Table II. The differences in lectin-binding patterns in SGC-7901, BGC-823 and MGC-803 cells.

\begin{tabular}{rlrrr}
\hline ID & \multicolumn{1}{c}{ Lectin } & SGC-7901 & BGC-823 & MGC-803 \\
\hline 1 & ConA $^{\mathrm{a}}$ & 10000 & 10000 & 10000 \\
2 & LCA & 3440 & 6131 & 8317 \\
3 & MAL-I & 347 & 1411 & 6100 \\
4 & LEL & 1297 & 2696 & 5634 \\
5 & Black bean crude & 3212 & 6916 & 8838 \\
6 & LcH & 5089 & 8099 & 12834 \\
7 & MAL-II & 3694 & 5937 & 7441 \\
8 & Jacalin & 571 & 1159 & 2469 \\
9 & PSA & 7047 & 7898 & 12174 \\
10 & UEA-I & 6455 & 1545 & 241 \\
11 & AAL & 8147 & 3991 & 3415 \\
\hline
\end{tabular}

${ }^{\mathrm{a} C o n A}$ was used as the normalized standard.

Differential $\alpha 2,3$-sialyltransferases expression in GC cells. The $\alpha 2,3$-sialyltransferases mediate the transfer of sialic acid with $\alpha 2,3$-linkage to terminal Gal residues. An examination of mRNA expression for $\alpha 2,3$-sialyltransferases in GC cell lines was carried out by real-time RT-PCR. The level of ST3Gal-IV mRNA in MGC-803 cells was significantly increased compared with that in SGC-7901 cells $(\mathrm{P}<0.05)$, whereas the mRNA levels of other sialyltransferases were not increased (Fig. 3A). Furthermore, the protein expression of ST3Gal-IV was also considerably enhanced in MGC-803 cells (Fig. 3B). These results suggest that the overexpression of $\alpha 2-3$ Sia may result from the upregulation of ST3Gal-IV in GC cells.

ST3Gal-IV mediates the migration and invasion of GC cells by regulation the formation of $\alpha 2-3$ Sia. To determine whether ST3Gal-IV promotes the migration and invasion potential of GC cells, we carried out the experiments in two parts: i) SGC-7901 cells were transfected with ST3Gal-IV overexpressing or control plasmid, and ii) MGC-803 cells were transfected with ST3Gal-IV or negative siRNA. Transfection efficiency was assessed using fluorescence microscopy (Fig. 4A). We observed that the mRNA and protein levels of ST3Gal-IV were markedly increased in SGC-7901 cells transiently transfected with ST3Gal-IV overexpressing plasmids. Inversely, real-time RT-PCR and western blot assays demonstrated that ST3Gal-IV expression was markedly decreased in MGC-803 cells after targeted siRNA treatment (Fig. 4B and C). Then the invasion and migration abilities of the cells were analyzed. As expected, the results showed that ectopic expression of ST3Gal-IV significantly enhanced the migration and invasion of SGC-7901 cells $(\mathrm{P}<0.05)$, whereas knockdown of ST3Gal-IVs markedly suppressed the migration and invasion of MGC-803 cells $(\mathrm{P}<0.05)$ (Fig. 5).

To confirm whether the expression of ST3Gal-IV was directly involved in the formation of $\alpha 2-3 \mathrm{Sia}$, lectin blot and flow cytometry assays were performed. We observed that the content of $\alpha 2-3$ Sia was increased by overexpression of ST3Gal-IV in SGC-7901 cells (Fig. 6). The opposite

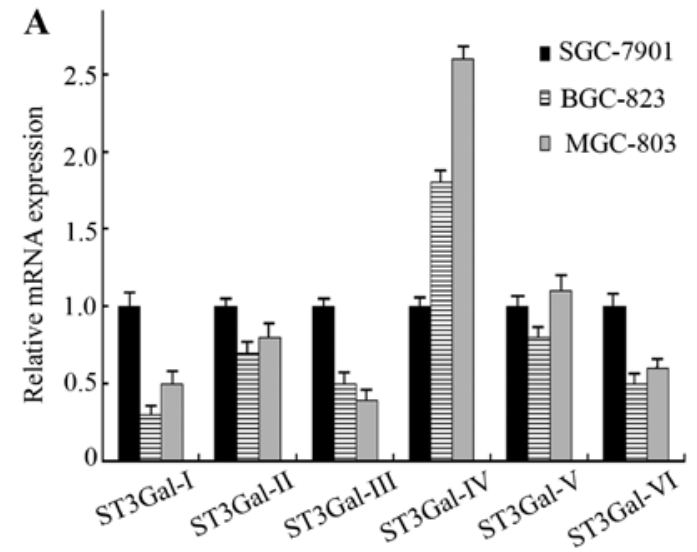

B

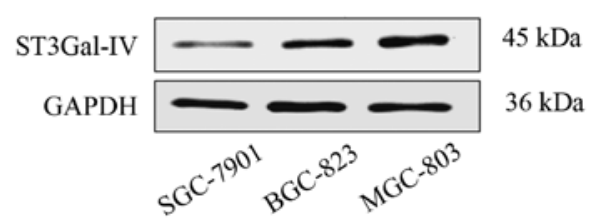

Figure 3. The expression of $\alpha 2,3$-sialyltransferases in GC cell lines. (A) The mRNA levels of $\alpha 2,3$-sialyltransferases were determined by real-time RT-PCR. (B) The protein expression levels of ST3Gal-IV were examined by western blot analysis.

results were obtained after ST3Gal-IV was knocked down in MGC-803 cells. The expression levels of ST3Gal-IV were positively associated with $\alpha 2-3 \mathrm{Sia}$ expression. Thus, we concluded that ST3Gal-IV significantly promoted the invasion and metastasis of GC cells via regulating the glycosylation profile in terms of $\alpha 2-3 \mathrm{Sia}$ chains.

Overexpression of $\alpha 2-3$ Sia is associated with adverse clinical characteristics and poor GC patient survival. We investigated the expression of $\alpha 2-3$ Sia in 80 paraffin-embedded GC tissues using lectin histochemistry. As shown in Fig. 7A, $\alpha 2-3$ Sia was mainly located in the membrane and cytoplasm of tumor cells. Thirty-six patients possessed low $\alpha 2-3$ Sia expression while the other 44 had high $\alpha 2-3$ Sia expression based on the IRS scores. The associations between $\alpha 2-3$ Sia expression and clinicopathological parameters are summarized in Table III. High $\alpha 2-3$ Sia expression was positively correlated with lymph node metastasis $(\mathrm{P}<0.001)$, TNM stage $(\mathrm{P}<0.001)$ and depth of tumor invasion $(\mathrm{P}=0.003)$. The results also showed that no significant correlation was observed between the $\alpha 2-3$ Sia expression and age, gender, tumor size, Lauren classification or differentiation.

The relationship between $\alpha 2-3 \mathrm{Sia}$ expression and the survival time of GC patients was analyzed with Kaplan-Meier survival analysis. We found that the overall survival time of high $\alpha 2-3$ Sia expression group was significantly shorter than that of low $02-3$ Sia expression group $(\mathrm{P}<0.05)$ (Fig. 7B). Moreover, univariate analysis in GC tissues showed that lymph node metastasis $(\mathrm{P}=0.004)$, TNM stage $(\mathrm{P}=0.008)$, depth of tumor invasion $(\mathrm{P}=0.034)$, and $\alpha 2-3$ Sia expression $(\mathrm{P}=0.002)$ were prognostic factors of overall survival (Table IV). Multivariate analysis using the Cox's proportional hazards model identified that overexpression of $\alpha 2-3 \mathrm{Sia}$ was an independent factor influencing OS (hazard ratio, 2.054; 95\% CI, 1.302-3.239; $\mathrm{P}=0.001$ ) of patients receiving curative resection for GC. 
Table III. Relationship between $\alpha 2-3$ Sia expression and clinicopathological features of GC patients.

\begin{tabular}{|c|c|c|c|c|}
\hline \multirow[b]{2}{*}{$\begin{array}{l}\text { Clinicopathological } \\
\text { features }\end{array}$} & \multirow[b]{2}{*}{$\begin{array}{l}\text { No. of } \\
\text { patients }\end{array}$} & \multicolumn{2}{|c|}{$\alpha 2-3$ Sia expression } & \multirow[b]{2}{*}{ P-value } \\
\hline & & $\begin{array}{c}\text { Low } \\
(\mathrm{n}=36)\end{array}$ & $\begin{array}{l}\text { High } \\
(\mathrm{n}=44)\end{array}$ & \\
\hline \multicolumn{5}{|l|}{ Age (years) } \\
\hline$<60$ & 32 & 13 & 19 & 0.521 \\
\hline$\geq 60$ & 48 & 23 & 25 & \\
\hline \multicolumn{5}{|l|}{ Gender } \\
\hline Male & 57 & 28 & 29 & 0.243 \\
\hline Female & 23 & 8 & 15 & \\
\hline \multicolumn{5}{|l|}{ Tumor size } \\
\hline$<5 \mathrm{~cm}$ & 34 & 19 & 15 & 0.093 \\
\hline$\geq 5 \mathrm{~cm}$ & 46 & 17 & 29 & \\
\hline \multicolumn{5}{|l|}{ Lauren classification } \\
\hline Intestinal & 50 & 24 & 26 & 0.087 \\
\hline Diffuse & 30 & 12 & 18 & \\
\hline \multicolumn{5}{|l|}{ Differentiation } \\
\hline Moderate-High & 54 & 26 & 28 & 0.153 \\
\hline Low & 26 & 10 & 16 & \\
\hline \multicolumn{5}{|l|}{ Invasion depth } \\
\hline $\mathrm{T} 1+\mathrm{T} 2$ & 15 & 12 & 3 & $0.003^{\mathrm{a}}$ \\
\hline $\mathrm{T} 3+\mathrm{T} 4$ & 65 & 24 & 41 & \\
\hline \multicolumn{5}{|c|}{ Lymph node metastasis } \\
\hline Positive & 20 & 16 & 4 & $0.000^{\mathrm{a}}$ \\
\hline Negative & 60 & 20 & 40 & \\
\hline \multicolumn{5}{|l|}{ TNM stage } \\
\hline $\mathrm{I}+\mathrm{II}$ & 32 & 26 & 6 & $0.000^{\mathrm{a}}$ \\
\hline $\mathrm{III}+\mathrm{IV}$ & 48 & 10 & 38 & \\
\hline
\end{tabular}

${ }^{\mathrm{a}} \mathrm{P}<0.05$ was considered significant.

\section{Discussion}

GC is a common malignancy worldwide, with a poor prognosis and low survival rates. Metastasis is a crucial factor in determining the prognosis of GC patients (4). Therefore, it is urgent to identify metastatic factors and elucidate the underlying molecular mechanisms. Recent studies revealed that abnormal changes of glycans in both structures and amounts are closely associated with tumor-cell migration, adhesion and metastasis $(16,17)$. In addition, altered glycosylation is a common feature of many types of cancers, including GC. Based on our data, we speculate that increased $\alpha 2-3$ Sia expression could be very important as indicator and functional contributor of GC metastasis and poor prognosis.

Lectin microarray has been proved as a useful high-throughput tool to analyze cell-surface glycans (18). For specific recognition, lectins reversibly bind to glycans and do not modify the structure of glycans. This technique has been successfully applied for screening cell-specific and functionally significant glycan markers. With nearly 100 different
A

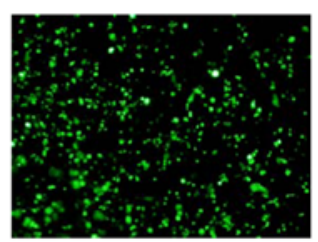

ST3Gal-IV plasmid

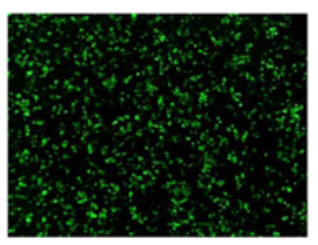

ST3Gal-IV siRNA

B

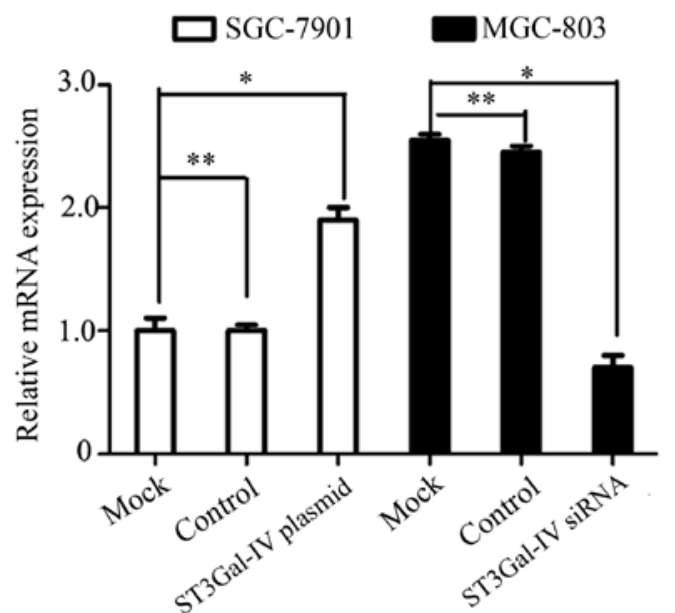

C

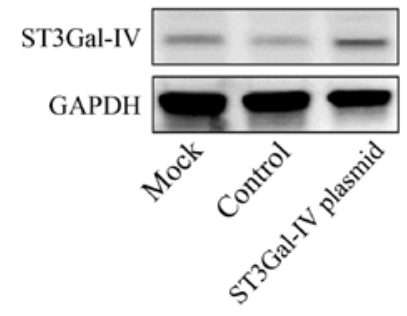

SGC-7901

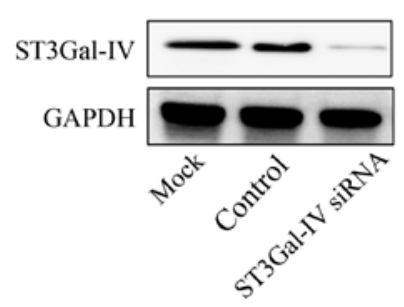

MGC-803
Figure 4. The expression of ST3Gal-IV in different groups. SGC-7901 and MGC-803 cells were transiently transfected with pEGFP-C1, pEGFP-C1-ST3Gal-IV plasmids, or negative siRNA, ST3Gal-IV siRNA, respectively. (A) Detection of transfection efficiency using fluorescence microscopy (x100). (B) The mRNA levels of ST3Gal-IV were determined by real-time RT-PCR. (C) The protein expression levels of ST3Gal-IV were examined by western blot analysis ( $\mathrm{*}<0.05$ and $\left.^{* *} \mathrm{P}>0.05\right)$.

arrayed lectins, such highly multiplexed characterization enabled the possibility for the detection of differences between cells and the identification of glycans responsible for these differences (19). Using the lectin microarray, Zhou et al confirmed that glycans containing a single terminal galactose in triple-negative breast cancer cells were proportional to their metastatic capacity (20). Nakajima et al reported that core $1 \mathrm{O}$-glycans exhibited strikingly intense signals in the cytoplasm and apical surfaces of colorectal cancer cells (21). Fry et al showed that the application of this technology was able to discover of specific glycan signatures associated with metastatic breast cancer (22). With a unique high-throughput lectin microarray, we identified clear differences in glycan composition between GC cells that differed in metastatic capacity. Three lectins, LEL, Jacalin and MAL-I displayed significant increases in binding in high-metastatic compared with low-metastatic GC cells. LEL specifically recognizes the polylactosamine-type $\mathrm{N}$-glycans (23). Jacalin strongly binds with $\mathrm{T}$ antigen (24). This is consistent with previous study 
Table IV. Univariate and multivariate Cox regression analyses of overall survival in GC patients.

\begin{tabular}{lccccr}
\hline & \multicolumn{2}{c}{ Univariate analysis } & & \multicolumn{2}{c}{ Multivariate analysis } \\
\cline { 2 - 3 } Variable & HR $(95 \% \mathrm{CI})$ & & Pr $(95 \% \mathrm{CI})$ & P-value \\
\hline Gender & $1.003(0.744-1.351)$ & 0.985 & & \\
Age (years) & $1.218(0.887-1.671)$ & 0.223 & & \\
Tumor size & $1.489(1.074-2.064)$ & 0.169 & & \\
Lauren classification & $1.237(0.974-1.688)$ & 0.145 & & \\
Differentiation & $1.096(0.785-1.952)$ & 0.753 & & & \\
Invasion depth & $1.549(1.033-2.322)$ & $0.034^{\mathrm{a}}$ & & $1.336(0.971-1.839)$ & $0.002^{\mathrm{a}}$ \\
Lymph node metastasis & $1.943(1.239-3.047)$ & $0.004^{\mathrm{a}}$ & & $1.378(0.959-1.979)$ & $0.005^{\mathrm{a}}$ \\
TNM stage & $1.590(1.132-2.233)$ & $0.008^{\mathrm{a}}$ & & $0.942(0.893-1.497)$ & $0.001^{\mathrm{a}}$ \\
Expression & $1.974(1.381-2.822)$ & $0.002^{\mathrm{a}}$ & & $2.054(1.302-3.239)$ & \\
\hline
\end{tabular}

${ }^{\mathrm{a}} \mathrm{P}<0.05$ was considered significant.

A

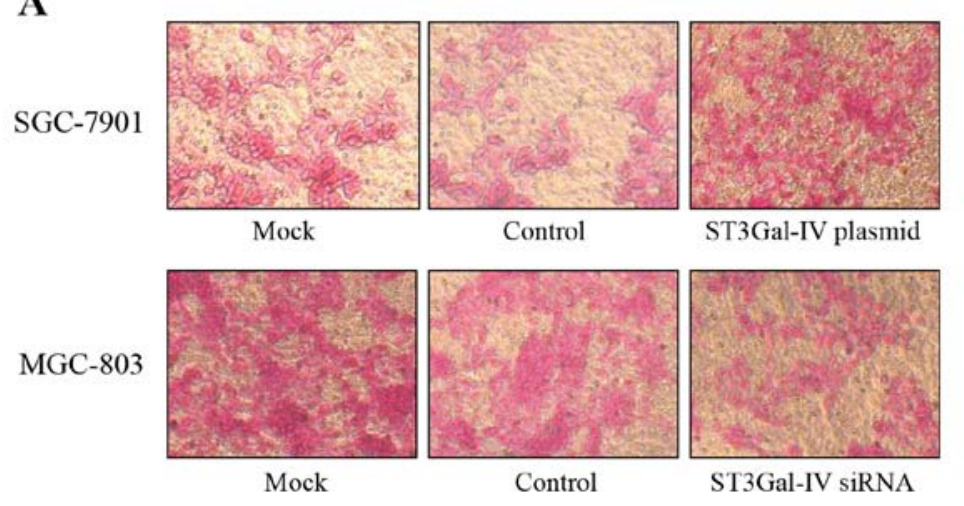

B

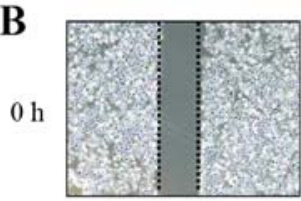

Mock

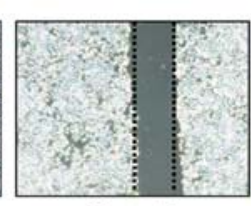

Control

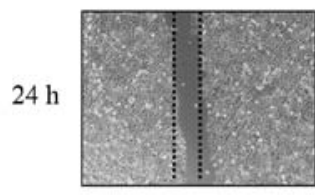

Mock

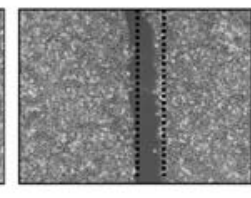

Control

SGC-7901

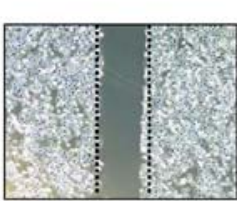

ST3Gal-IV plasmid

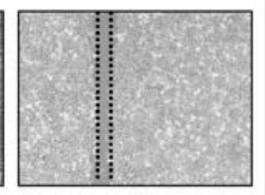

ST3Gal-IV plasmid

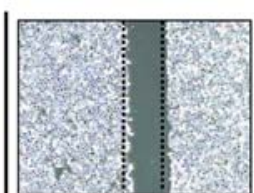

Mock

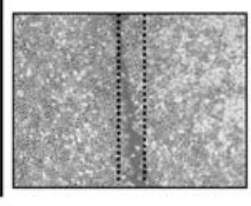

Mock

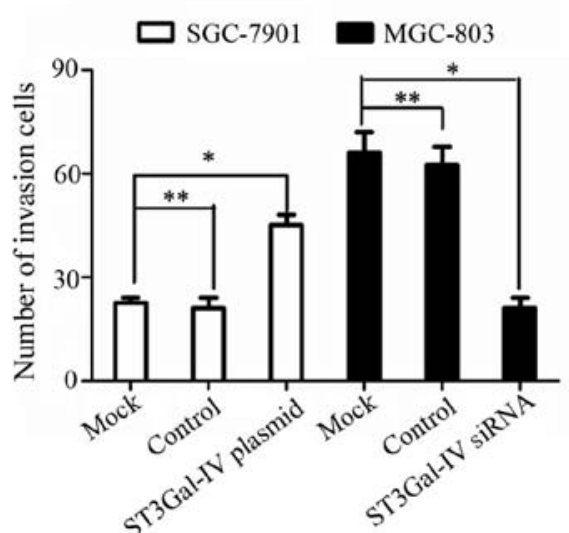

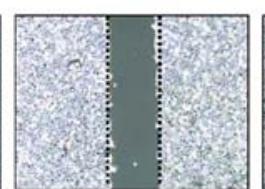

Control

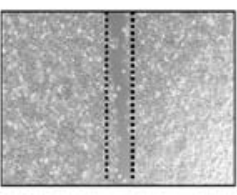

Control

MGC-803

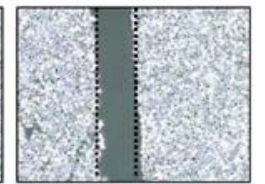

ST3Gal-IV siRNA

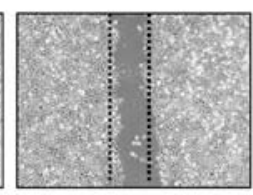

ST3Gal-IV siRNA

Figure 5. The effects of ST3Gal-IV on invasion and migration of GC cell lines. SGC-7901 and MGC-803 cells were transiently transfected with pEGFP-C1, pEGFP-C1- ST3Gal-IV plasmids, or negative siRNA, ST3Gal-IV siRNA, respectively. (A) The invasive ability was evaluated by Transwell assay ("P<0.05 and $\left.{ }^{* *} \mathrm{P}>0.05\right)$. (B) The migratory ability was evaluated with a wound-healing assay.

which suggested that highly metastatic cancer cells expressed more polylactosamine chains (25) and $\mathrm{T}$ antigen expression was related to the depth of invasion and lymph node metastasis in GC patients (26). MAL-I is used widely in the detection and characterization of $\alpha 2-3 \mathrm{Sia}$ in human cancer cells (15). While the functional implications of these changes remains to be further investigated, the present study revealed the important role of $\alpha 2-3$ Sia signal in regulating GC cell migration and invasion.

Sialic acids, as terminal monosaccharide were added to glycoproteins or glycolipids, could link to Gal $\beta 1,3(4) \mathrm{GlcNAc} / \mathrm{Glc}$ via $\alpha 2,3$ or $\alpha 2,6$ and mediated a variety of pathological process (27). The altered sialic acid residues were reported to be closely associated with cellular adhesion, migration and metastasis in 

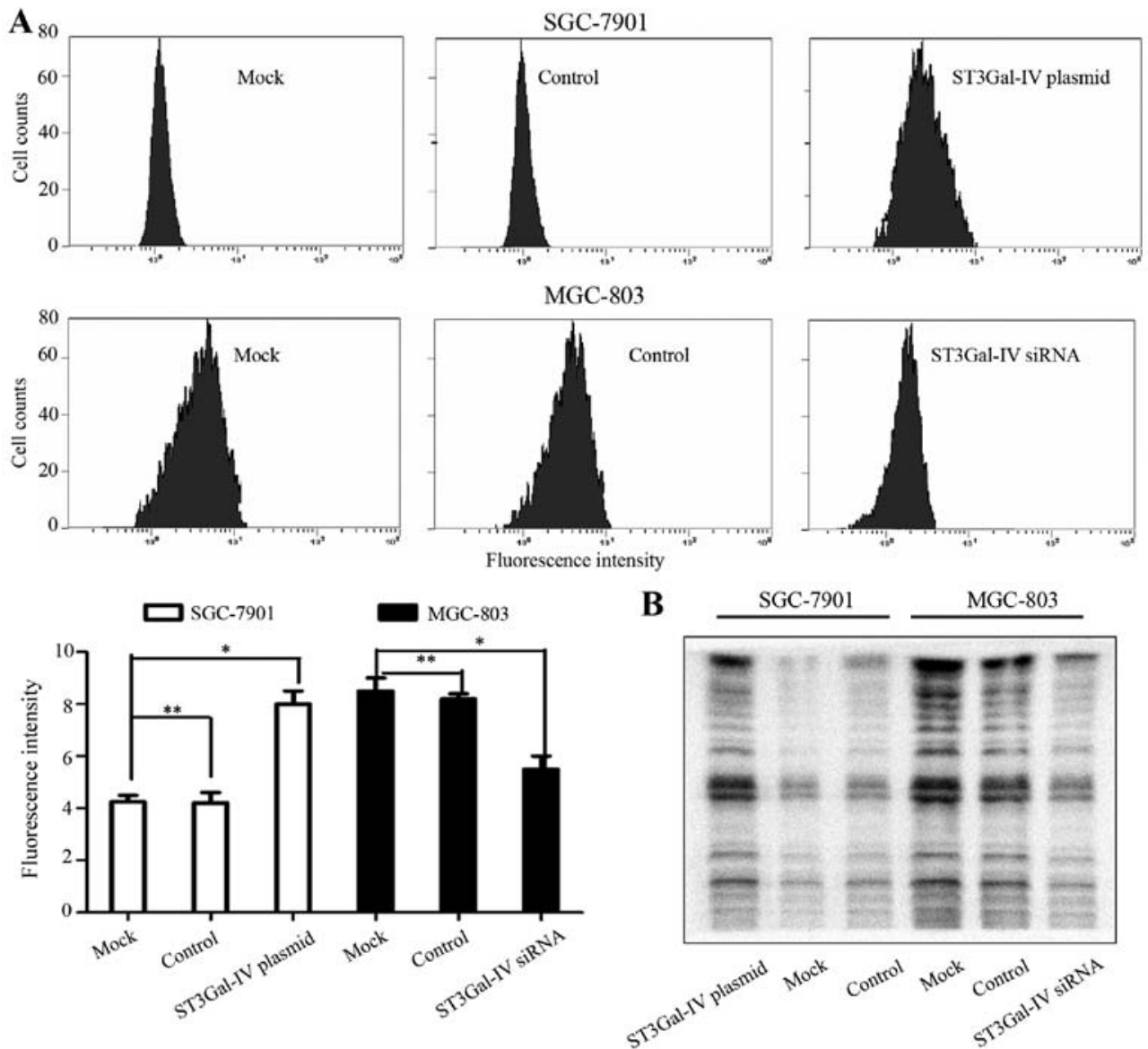

Figure 6. The effects of ST3Gal-IV on the formation of $\alpha 2-3$ Sia. SGC-7901 and MGC-803 cells were transiently transfected with pEGFP-C1,pEGFP-C1-ST3Gal-IV plasmids, or negative siRNA, ST3Gal-IV siRNA, respectively. (A) Results of the flow cytometric analysis. Histograms of fluorescence intensities of cells with specific glycan expression. (B) Results of the lectin blot analysis.

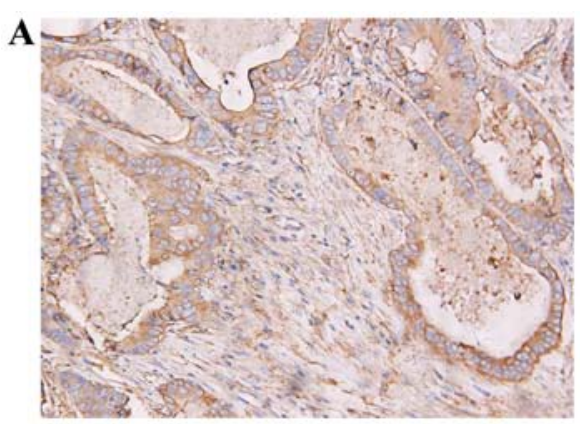

High expression of $\alpha 2-3 \mathrm{Sia}$

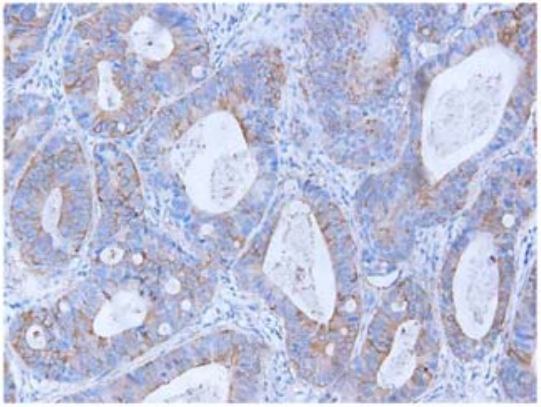

Low expression of $\alpha 2-3$ Sia

B

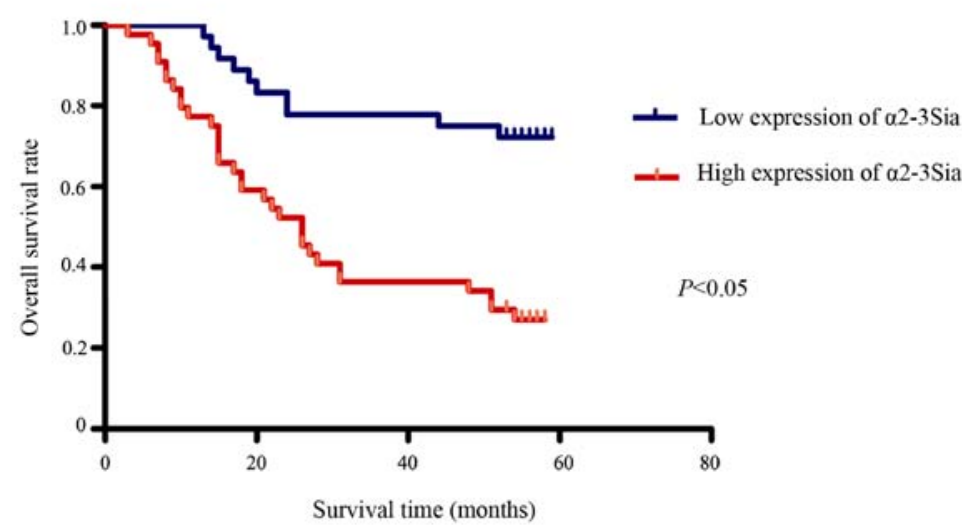

Figure 7. The expression of $\alpha 2-3$ Sia in GC tissues, and survival curve of patients. (A) Characterization of $\alpha 2-3$ Sia expression in human GC tissues by immunohistochemistry staining (x200). (B) Overall survival curves for $80 \mathrm{GC}$ patients, grouped according to $\alpha 2-3$ Sia expression $(\mathrm{P}<0.05$ ). 
tumor cells (28-30). Up to now, an increasing number of studies suggested that an increased sialic acid content in cancer cells is caused by upregulating of sialyltransferase and depended on the mRNA levels of sialyltransferase gene. ST3Gal-I is responsible for $\alpha 2,3$-sialylation of Gal $\alpha 1,3$ GalNAc on O-linked glycans. Transfer of $\alpha 2-3$ Sia to Gal $\alpha 1$, (3)4GlcNAc on N-linked glycans is catalyzed by ST3Gal-III or ST3Gal-IV (31). Increased expression, relative to normal tissue, of ST3Gal-IV is observed in many carcinomas, especially GC (32). In agreement with these studies, we found a significantly increased expression of ST3Gal-IV in highly metastatic GC cells. Moreover, we have shown that there is a significant correlation between ST3Gal-IV expression and $\alpha 2-3$ Sia expression in GC cells. Further functional study will be conducted to explore the correlation between $\alpha 2-3 \mathrm{Sia}$ and ST3Gal-III in other GC cells. More types of GC cells will be used and the role of ST3Gal-III will be investigated in our future study.

The molecular mechanisms underlying the effect of $\alpha 2-3$ Sia on the invasion and metastasis of human GC were largely unknown. In this study, we first examined whether ST3Gal-IV could affect cells migration and invasion ability by wound-healing assay and Transwell assays. The results showed that suppression of ST3Gal-IV in MGC-803 cells led to significant decreases in cell migration and invasion, while its upregulation in SGC-7901 cells resulted in the converse. In addition, the levels of $\alpha 2-3 \mathrm{Sia}$ residues were subsequently changed by the alteration of ST3Gal-IV. These observations clearly indicate that the changes in ST3Gal-IV expression levels may have impact in the remodeling of cell surface sialylation, which may consequently affect the biological functions of tumor cells such as invasion and metastasis.

ST3Gal-IV was reported to be important in invasion and metastasis of tumor cells. For example, Soyasaponin-1 inhibited ST3Gal-IV activity and decreased $\alpha 2-3 \mathrm{Sia}$ in MCF-7 breast cancer cells (33). ST3Gal-IV was also overexpressed in human hepatic carcinoma cell lines, compared with normal hepatic cell line (34). ST3Gal-IV was involved in key steps of pancreatic tumor progression processes and was highly expressed in most pancreatic adenocarcinoma tissues (35). Moreover, overexpression of ST3Gal-IV could induce activation of cell signaling pathways and alteration in GC cell line phenotype (36). TNF-induced upregulation of the ST3Gal-IV transcript was mediated by MSK1/2 through the ERK and p38 MAPK pathways (37). These data may offer important clues for future studies into the mechanism of $\alpha 2-3$ Sia related to the progression and metastasis of GC.

The results of the present study also showed that $\alpha 2-3 \mathrm{Sia}$ expression was positively correlated with lymph node metastasis, TNM stage and depth of tumor invasion. Similar results were also observed by Wang et al (12). Interestingly, KaplanMeier analysis revealed that $\alpha 2-3$ Sia expression predicted poor overall survival. Multivariate analysis using the Cox's proportional hazards model suggested $\alpha 2-3$ Sia expression was an independent prognostic indicator of patients' overall survival. To our knowledge, our results for the first time provide the above new evidence of $\alpha 2-3$ Sia in the prognosis of $\mathrm{GC}$ patients.

In conclusion, we found several lectins that exhibited altered bindings to GC cells with different metastatic abilities using a high-density lectin microarray. In particular, the levels of $\alpha 2-3$ Sia, which can be regulated by ST3Gal-IV, showed a positive correlation with the metastatic capacity of GC cells. Overexpression of $\alpha 2-3 \mathrm{Sia}$ could be an independent factor predicting poor survival and an attractive therapeutic target for GC, but further studies are required.

\section{Acknowledgements}

The present study was supported by the Natural Science Foundation of Hubei Provincial Department of Education (no. Q20162115), Innovative Research Team of Hubei University of Medicine (no. 2014CXG02), and the Scientific and Technological Project of Shiyan City of Hubei Province (no. 15K65).

\section{References}

1. Wang C, Zhang J, Cai M, Zhu Z, Gu W, Yu Y and Zhang X: DBGC: A Database of Human Gastric Cancer. PLoS One 10: e0142591, 2015.

2. Torre LA, Bray F, Siegel RL, Ferlay J, Lortet-Tieulent J and Jemal A: Global cancer statistics, 2012. CA Cancer J Clin 65: 87-108, 2015

3. Chen W, Zheng R, Baade PD, Zhang S, Zeng H, Bray F, Jemal A, Yu XQ and He J: Cancer statistics in China, 2015. CA Cancer J Clin 66: 115-132, 2016.

4. Liu X, Ge X, Zhang Z, Zhang X, Chang J, Wu Z, Tang W, Gan L, Sun M and Li J: MicroRNA-940 promotes tumor cell invasion and metastasis by downregulating ZNF24 in gastric cancer. Oncotarget 6: 25418-25428, 2015.

5. Hart GW and Copeland RJ: Glycomics hits the big time. Cell 143: 672-676, 2010.

6. Munkley J and Elliott DJ: Hallmarks of glycosylation in cancer. Oncotarget 7: 35478-35489, 2016.

7. Pinho SS and Reis CA: Glycosylation in cancer: Mechanisms and clinical implications. Nat Rev Cancer 15: 540-555, 2015.

8. Tsai CH, Tzeng SF, Chao TK, Tsai CY, Yang YC, Lee MT, Hwang JJ, Chou YC, Tsai MH, Cha TL, et al: Metastatic progression of prostate cancer is mediated by autonomous binding of galectin-4-O-glycan to cancer cells. Cancer Res 76: 5756-5767, 2016.

9. Zhang X, Wang Y, Qian Y, Wu X, Zhang Z, Liu X, Zhao R, Zhou L, Ruan Y, Xu J, et al: Discovery of specific metastasisrelated $\mathrm{N}$-glycan alterations in epithelial ovarian cancer based on quantitative glycomics. PLoS One 9: e87978, 2014.

10. Liu L, Yan B, Huang J, Gu Q, Wang L, Fang M, Jiao J and Yue X: The identification and characterization of novel N-glycan-based biomarkers in gastric cancer. PLoS One 8: e77821, 2013.

11. Zhao YP, Xu XY, Fang M, Wang H, You Q, Yi CH, Ji J, Gu X, Zhou PT, Cheng C, et al: Decreased core-fucosylation contributes to malignancy in gastric cancer. PLoS One 9: e94536, 2014.

12. Wang FL, Cui SX, Sun LP, Qu XJ, Xie YY, Zhou L, Mu YL, Tang W and Wang YS: High expression of alpha 2,3-linked sialic acid residues is associated with the metastatic potential of human gastric cancer. Cancer Detect Prev 32: 437-443, 2009.

13. Shen L, Yu M, Xu X, Gao L, Ni J, Luo Z and Wu S: Knockdown of $\beta 3 \mathrm{GnT} 8$ reverses 5-fluorouracil resistance in human colorectal cancer cells via inhibition the biosynthesis of polylactosamine-type N-glycans. Int J Oncol 45: 2560-2568, 2014.

14. Wang X, He H, Zhang H, Chen W, Ji Y, Tang Z, Fang Y, Wang C, Liu F, Shen Z, et al: Clinical and prognostic implications of $\beta 1$, $6-\mathrm{N}$-acetylglucosaminyltransferase $\mathrm{V}$ in patients with gastric cancer. Cancer Sci 104: 185-193, 2013.

15. Badr HA, Elsayed AI, Ahmed H, Dwek MV, Li CZ and Djansugurova LB: Preferential lectin binding of cancer cells upon sialic acid treatment under nutrient deprivation. Appl Biochem Biotechnol 171: 963-974, 2013.

16. Nie H, Liu X, Zhang Y, Li T, Zhan C, Huo W, He A, Yao Y, Jin Y, $\mathrm{Qu} \mathrm{Y,} \mathrm{et} \mathrm{al:} \mathrm{Specific} \mathrm{N-glycans} \mathrm{of} \mathrm{hepatocellular} \mathrm{carcinoma} \mathrm{cell}$ surface and the abnormal increase of core- $\alpha-1,6$-fucosylated triantennary glycan via N-acetylglucosaminyltransferases-IVa regulation. Sci Rep 5: 16007, 2015.

17. Häuselmann I and Borsig L: Altered tumor-cell glycosylation promotes metastasis. Front Oncol 4: 28, 2014. 
18. Hirabayashi J, Kuno A and Tateno H: Development and applications of the lectin microarray. Top Curr Chem 367: 105-124, 2015.

19. Tao SC, Li Y, Zhou J, Qian J, Schnaar RL, Zhang Y, Goldstein IJ, Zhu H and Schneck JP: Lectin microarrays identify cell-specific and functionally significant cell surface glycan markers. Glycobiology 18: 761-769, 2008.

20. Zhou SM, Cheng L, Guo SJ, Wang Y, Czajkowsky DM, Gao H, Hu XF and Tao SC: Lectin RCA-I specifically binds to metastasisassociated cell surface glycans in triple-negative breast cancer. Breast Cancer Res 17: 36, 2015.

21. Nakajima K, Inomata M, Iha H, Hiratsuka T, Etoh T, Shiraishi N, Kashima $\mathrm{K}$ and Kitano S: Establishment of new predictive markers for distant recurrence of colorectal cancer using lectin microarray analysis. Cancer Med 4: 293-302, 2015.

22. Fry SA, Afrough B, Lomax-Browne HJ, Timms JF, Velentzis LS and Leathem AJ: Lectin microarray profiling of metastatic breast cancers. Glycobiology 21: 1060-1070, 2011.

23. Mitsui Y, Yamada K, Hara S, Kinoshita M, Hayakawa T and Kakehi K: Comparative studies on glycoproteins expressing polylactosamine-type $\mathrm{N}$-glycans in cancer cells. J Pharm Biomed Anal 70: 718-726, 2012.

24. Ayaz Ahmed KB, Mohammed AS and Veerappan A: Interaction of sugar stabilized silver nanoparticles with the T-antigen specific lectin, jacalin from Artocarpus integrifolia. Spectrochim Acta A Mol Biomol Spectrosc 145: 110-116, 2015.

25. Kinoshita M, Mitsui Y, Kakoi N, Yamada K, Hayakawa T and Kakehi K: Common glycoproteins expressing polylactosaminetype glycans on matched patient primary and metastatic melanoma cells show different glycan profiles. J Proteome Res 13 1021-1033, 2014

26. Carneiro F, David L and Sobrinho-Simões M: Prognostic significance of $\mathrm{T}$ antigen expression in patients with gastric carcinoma. Cancer 78: 2448-2450, 1996.

27. Cui H, Lin Y, Yue L, Zhao X and Liu J: Differential expression of the $\alpha 2,3$-sialic acid residues in breast cancer is associated with metastatic potential. Oncol Rep 25: 1365-1371, 2011.

28. Schultz MJ, Swindall AF and Bellis SL: Regulation of the metastatic cell phenotype by sialylated glycans. Cancer Metastasis Rev 31: 501-518, 2012.
29. Liu YC, Yen HY, Chen CY, Chen CH, Cheng PF, Juan YH, Chen $\mathrm{CH}$, Khoo KH, Yu CJ, Yang PC, et al: Sialylation and fucosylation of epidermal growth factor receptor suppress its dimerization and activation in lung cancer cells. Proc Natl Acad Sci USA 108: 11332-11337, 2011

30. Wang S, Chen X, Wei A, Yu X, Niang B and Zhang J: $\alpha 2,6$-linked sialic acids on $\mathrm{N}$-glycans modulate the adhesion of hepatocarcinoma cells to lymph nodes. Tumour Biol 36: 885-892, 2015.

31. Petretti T, Kemmner W, Schulze B and Schlag PM: Altered mRNA expression of glycosyltransferases in human colorectal carcinomas and liver metastases. Gut 46: 359-366, 2000.

32. Petretti T, Schulze B, Schlag PM and Kemmner W: Altered mRNA expression of glycosyltransferases in human gastric carcinomas. Biochim Biophys Acta 1428: 209-218, 1999.

33. Higai K,Miyazaki N,Azuma Y and MatsumotoK:Interleukin-1beta induces sialyl Lewis X on hepatocellular carcinoma HuH-7 cells via enhanced expression of ST3Gal IV and FUT VI gene. FEBS Lett 580: 6069-6075, 2006.

34. Zhang Y, Zhao W, Zhao Y and He Q: Expression of ST3Gal, ST6Gal, ST6GalNAc and ST8Sia in human hepatic carcinoma cell lines, HepG-2 and SMMC-7721 and normal hepatic cell line, L-02. Glycoconj J 32: 39-47, 2015.

35. Pérez-Garay M, Arteta B, Llop E, Cobler L, Pagès L, Ortiz R, Ferri MJ, de Bolós C, Figueras J, de Llorens R, et al: $\alpha 2,3$-Sialyltransferase ST3Gal IV promotes migration and metastasis in pancreatic adenocarcinoma cells and tends to be highly expressed in pancreatic adenocarcinoma tissues. Int J Biochem Cell Biol 45: 1748-1757, 2013.

36. Gomes C, Osorio H, Pinto MT and Celso A Reis: Overexpression of ST3Gal-IV induces activation of cell signaling pathways and alteration in gastric cancer cell line phenotype. Glycobiology 22: 1645-1646, 2012.

37. Colomb F, Vidal O, Bobowski M, Krzewinski-Recchi MA Harduin-Lepers A, Mensier E, Jaillard S, Lafitte JJ, Delannoy P and Groux-Degroote S: TNF induces the expression of the sialyltransferase ST3Gal IV in human bronchial mucosa via MSK1/2 protein kinases and increases FliD/sialyl-Lewis(x)-mediated adhesion of Pseudomonas aeruginosa. Biochem J 457: 79-87, 2014. 